\title{
Thermal Management in Plug-In Hybrid Electric Vehicles: a Real-Time Nonlinear Model Predictive Control Implementation
}

\author{
J. Lopez-Sanz, Carlos Ocampo-Martinez Senior Member, IEEE, Jesus Alvarez-Florez, Manuel \\ Moreno-Eguilaz, Rafael Ruiz-Mansilla, Julian Kalmus, Manuel Gräeber, Gerhard Lux
}

\begin{abstract}
A real-time nonlinear model predictive control (NMPC) for the thermal management (TM) of the electrical components cooling circuit in a Plug-In Hybrid Electric Vehicle (PHEV) is presented. The electrical components are highly temperature-sensitive and therefore working out of the ranges recommended by the manufacturer can lead to their premature aging or even failure. Consequently, the goals for an accurate and efficient TM are two: to keep the main component, the Li-ion battery, within optimal working temperatures, and to consume the minimum possible electrical energy through the cooling circuit actuators. This multi-objective requirement is formulated as a finite-horizon optimal control problem (OCP) that includes a multi-objective cost function, several constraints and a prediction model especially suitable for optimization. The associated NMPC is performed on real-time by the optimization package MUSCOD-II and is validated in three different repeatable test-drives driven with a PHEV. Starting from identical conditions, each cycle is driven once being the cooling
\end{abstract}

J. Lopez-Sanz and G. Lux are with Innovation and Alternative Mobility Department, SEAT Technical Center, Autovia A-2, Km. 585 Apdo. de Correos 91, 08760 Martorell, Spain, e-mails: extern.jorge.lopez@seat.es, gerhard.lux@seat.es

C. Ocampo-Martinez is with Automatic Control Department, Universitat Politècnica de Catalunya, Institut de Robòtica i Informàtica Industrial (CSIC-UPC), Llorens i Artigas, 4-6, 08028 Barcelona, Spain, e-mail: cocampo@iri.upc.edu

J. Alvarez-Florez is with the Center for Engines and Heat Installation Research (CREMIT), Technical University of Catalonia, Barcelona Tech., 08028 Barcelona, Spain, e-mail: jalvarez@mmt.upc.edu

M. Moreno-Eguilaz is with the Center Innovation Electronics, Motion Control and Industrial Applications (MCIA), Technical University of Catalonia, Barcelona Tech., 08028 Barcelona, Spain, email: manuel.moreno.eguilaz@upc.edu

R. Ruiz-Mansilla is with the Green Technologies Research Group (GREENTECH), Technical University of Catalonia, Barcelona Tech.,08028 Barcelona, Spain, e-mail: rafael.ruiz@upc.edu

J. Kalmus works at TLK-Thermo GmbH, Hans-Sommer-Str.5, 38106 Braunschweig, Germany, e-mail: j.kalmus@tlk-thermo.de

M. Gräber works at TLK Energy GmbH, Steppenbergweg 30, 52074 Aachen, Germany, e-mail: manuel.graeber@tlk-energy.de

This work was supported by the catalan Government: la Generalitat de Catalunya. Corresponding author: Jorge Lopez-Sanz extern.jorge.lopez@seat.es

Manuscript received XXX; revised XXX. circuit controlled with NMPC and once with a conventional approach based on a finite-state machine. Compared to the conventional strategy, the NMPC proposed here results in a more accurate and healthier temperature performance, and at the same time, leads to reductions in the electrical consumption up to $8 \%$.

Index Terms-nonlinear model predictive control (NMPC), thermal management, plug-in hybrid electric vehicles (PHEV), Li-ion battery cooling.

\section{INTRODUCTION}

$\mathbf{I}$ $\mathrm{N}$ electrified vehicles, an accurate TM of the electric traction components is crucial to avoid premature costly repairs and ensure safety and performance requirements [1]. Among them, the Li-ion battery package is the most critical due to its cost and its direct relation to the vehicle autonomy, which is definitely the electromobility market penetration bottleneck. Accurate TM solutions for Li-ion batteries are based usually on liquid cooling systems with complex pipes configurations that allow several options for heat dissipation. To control these circuits, multiple electrical actuators are needed. Since a misuse of electrical actuators contributes to a further decrease in vehicle autonomy, optimal control methods become quite attractive for accurate and efficient TM. Compared to the classical approach of using tuned Proportional-Integral-Derivative (PID) controllers according to a set of rules learned from experience, optimization-based methods such as NMPC exploit their potential in systems with:

- multiple inputs multiple outputs (MIMO).

- several goals that can be contradictory.

- numerous constraints that must be fulfilled, among others.

Although the many advantages, there are also some challenges for NMPC to spread in the automotive sector. The computational burden is one of them. A proof of this fact is the large number of existing offline applications in literature compared to the online category. Moreover, it 
is common that real-time capable NMPC applications are not validated directly in the real vehicle, but in a simpler context. This is the case of [2], where NMPC for adaptive cruise control is tested in a Hardware in the Loop (HIL) configuration on a dynamic engine test bench or [3], where an NMPC application for optimal trajectory generation in Long Heavy Vehicles Combinations that validated the controller in a motion simulator. In [4], the real-time NMPC strategy for an hybrid electric vehicle (HEV) power management is validated in simulations and the same is done in [5] to show the potential of NMPC for HEV fuel and emissions minimization. The validation through simulation/test bench environments in all these examples and many more is a necessary first step for every real-time application.

The purpose of this article is to use NMPC for the TM of the Li-ion battery (BAT) and the power electronics (PE) in a PHEV prototype. The validation of the feedback control designed by using the optimization tool MUSCOD-II [6] is done by means of a comparison to a finite-state machine control. The novelty of this paper is that the optimizer runs on an Intel ${ }^{\mathrm{R}} \mathrm{Core}^{\mathrm{TM}} \mathrm{i5-3320 \textrm {M }}$ Processor with the two cores operating at $2.6 \mathrm{GHz}$ and with $8 \mathrm{~GB}$ of RAM on real-time and overtakes the TM control by means of an electronic control unit (ECU) bypass performed on a rapid prototyping (RP) module. This NMPC implementation corresponds to a new step in the NMPC standardization road map suggested in Fig. 1 , where the final goal is to have the algorithm running embedded in the vehicle. In this sense, [7] points FPGA or multicore microprocessors as the suitable platforms to exploit parallelization of the NMPC controller design.

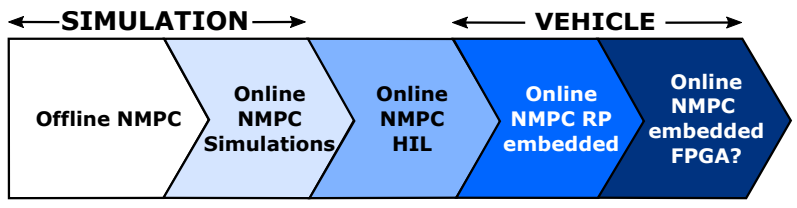

Fig. 1: NMPC roadmap in the automotive sector.

The remainder of this paper is structured as follows. Section II presents a brief description of the control plant. Section III gives an overview of the model, more extensively treated in [8], and defines the goals and constraints of the control problem. Section IV deals with the numerical solution of the NMPC problem. In Section V, the hardware implementation in the vehicle is presented and Section VI describes the driving scenarios in which validation took place. Finally, Section VII shows the results and the conclusions and final remarks are drawn in Section VIII.



Fig. 2: The studied cooling circuit.

\section{Problem Statement}

The cooling circuit to be controlled by NMPC can be seen in Fig. 2. The purpose of the circuit is to keep the BAT, PE and charger modules in the temperature regions that assure safety, suitable operation and reduce ageing caused by thermal stress. With this circuit, the heat generated in the electrical components due to the Joule Effect can be dissipated to the air or to the Air Conditioning (AC) circuit. Notice that:

- Only the driving situation is treated here, not the charging one. For this reason, the charger represents only a passive thermal mass in the circuit.

- The coolant is a water/glycol mixture and its possible paths are shown in the blue and black continuous lines in Fig. 2.

- The heat transfer with the air is done by means of a coolant/air heat exchanger, the cooler in Fig. 2.

- The heat transfer to the AC-circuit is done by a coolant/refrigerant heat-exchanger parallel to the evaporator called chiller in Fig. 2.

The heat transfer can be controlled through the coolant flow by six electrical actuators: two pumps, three solenoid valves and one fan, all in gray in Fig. 2. The control signals for these actuators are from the right top clockwise:

- Valve COOLER: Enables/disables the coolant flow through the cooler. With the value " 0 " the valve allows the cooler path, while " 1 " stands for the bypass.

- $P W M_{F A N}$ : The fan increases the air mass flow rate in front of the cooler and thus the heat exchange. 
It is controlled by a pulse width modulated (PWM) signal.

- $P W M_{P E}$ : The electrical pump before the PE is also governed by a PWM signal.

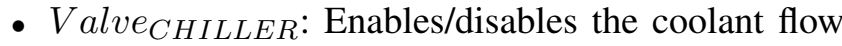
through the chiller. The value " 1 " is for chiller active, " 0 " stands for chiller inactive.

- Valve $C I R C U I T:$ Enables switching between $\mathrm{big} / \mathrm{small}$ circuit configurations. If Valve CIRCUIT is set to " 1 ", the big circuit configuration is active and the coolant flows through the charger, the cooler, the chiller, the BAT and the PE, consecutively. On the contrary, if Valve CIRCUIT is set to " 0 ", the coolant flows through two separate circuits: the BAT-chiller circuit and the charger-cooler-PE circuit. Consequently, in this mode, the heat transfer between the BAT-chiller and the PE-charger-cooler is disabled. Notice that to propel the coolant in two different separated circuits, two electric pumps are required.

- $P W M_{B A T}$ : The electrical pump in front of the chiller is also governed by a PWM signal.

As said in the introduction, the high number of electrical actuators offers an accurate TM but also supposes a challenge in efficiency: to spend as less electrical energy as possible. With the control methodology described in Section III, the aim is to formulate and solve this problem.

\section{Modeling And Optimal Control Problem FORMULATION}

The development of a system model is a crucial step for the NMPC strategy since it provides the predictive ability. The model of the cooling circuit in Fig. 2 is a system of ordinary differential equations (ODE) of the following general form:

$$
\dot{x}(t)=f(x(t), u(t), p)
$$

where $x \in \mathbb{R}^{\mathrm{n}_{\mathrm{x}}}$ represents the states of the plant, $u \in$ $\mathbb{R}^{\mathrm{n}_{u}}$ stands for the control inputs and $p \in \mathbb{R}^{\mathrm{n}_{\mathrm{p}}}$ for the time-invariant parameters. It is important to highlight that all the states $x$ are available from sensors equipped in the real vehicle.

Given the complexity and length of the mathematical model of the considered system, the reader can find its main lines in [8]. The interaction of the variables and constitutive elements of the resultant model are shown in Fig. 3. The model has been written in the software Dymola [9], which is based on the objectoriented language Modelica [10] and is a combination of physical equations and measurements stored in look-

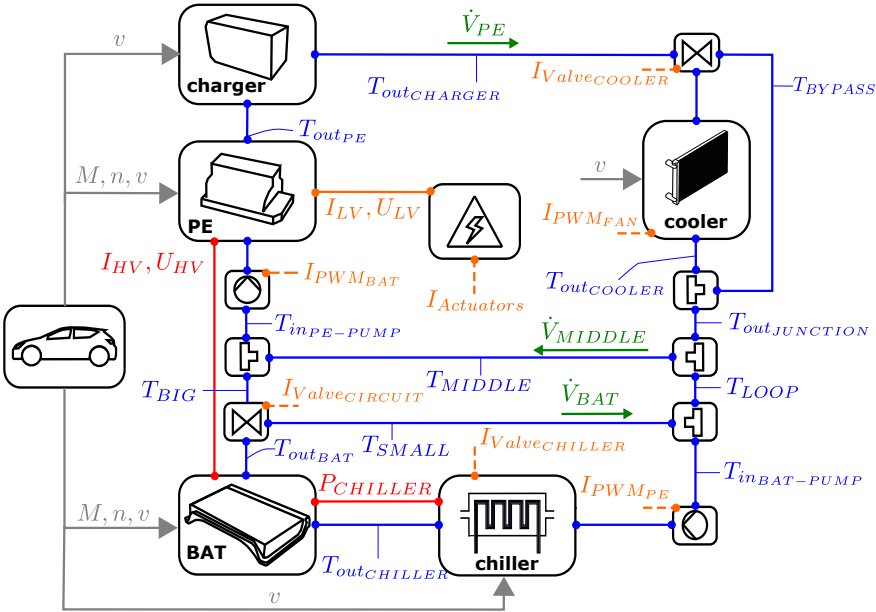

Fig. 3: Main variables and elements of the cooling circuit model developed in Modelica.

up tables that describe the cooling circuit behavior in multiple domains.

The physical equations of the model come mainly from energy balances. In the thermal domain, for instance, at each electric component the first thermodynamic law, (2), is applied to describe how the heat flow induced by the Joule effect $\dot{Q}_{\text {induced }}$ is dissipated in the coolant $\dot{Q}_{\text {coolant }}$, the ambient air $\dot{Q}_{\text {ambient }}$ and the component itself $\dot{Q}_{t h m}$, that is,

$$
\begin{aligned}
\frac{d U(t)}{d t} & =\dot{Q}_{t h m}(t) \\
& =\dot{Q}_{\text {induced }}(t)-\dot{Q}_{\text {ambient }}(t)-\dot{Q}_{\text {coolant }}(t) .
\end{aligned}
$$

The model consists of around 500 equations and 1300 variables that arise from the equations explicitly described inside the different submodels and the automatic generated connection equations. With the help of the model-export methodology described in [11], it is quite straightforward and error-free to pass the high number of equations to the MUSCOD-II.

To get an overview of the system states contained in the dynamic model, (3) corresponds with a condensed form of the model, where the relation between the variables used here and the control inputs can be found in [8].

Furthermore, to measure the performance of the system, a so called objective or cost function was developed. This cost function is an indirect measurement of the system performance. To this end, the performance indices of Fig. 4 are used to evaluate the TM in terms of accuracy and efficiency.

The cost term $c_{T}$ (on the left of Fig. 4) describes, with the following polynomial, the effect of the working temperature on the battery, so that the further from the 


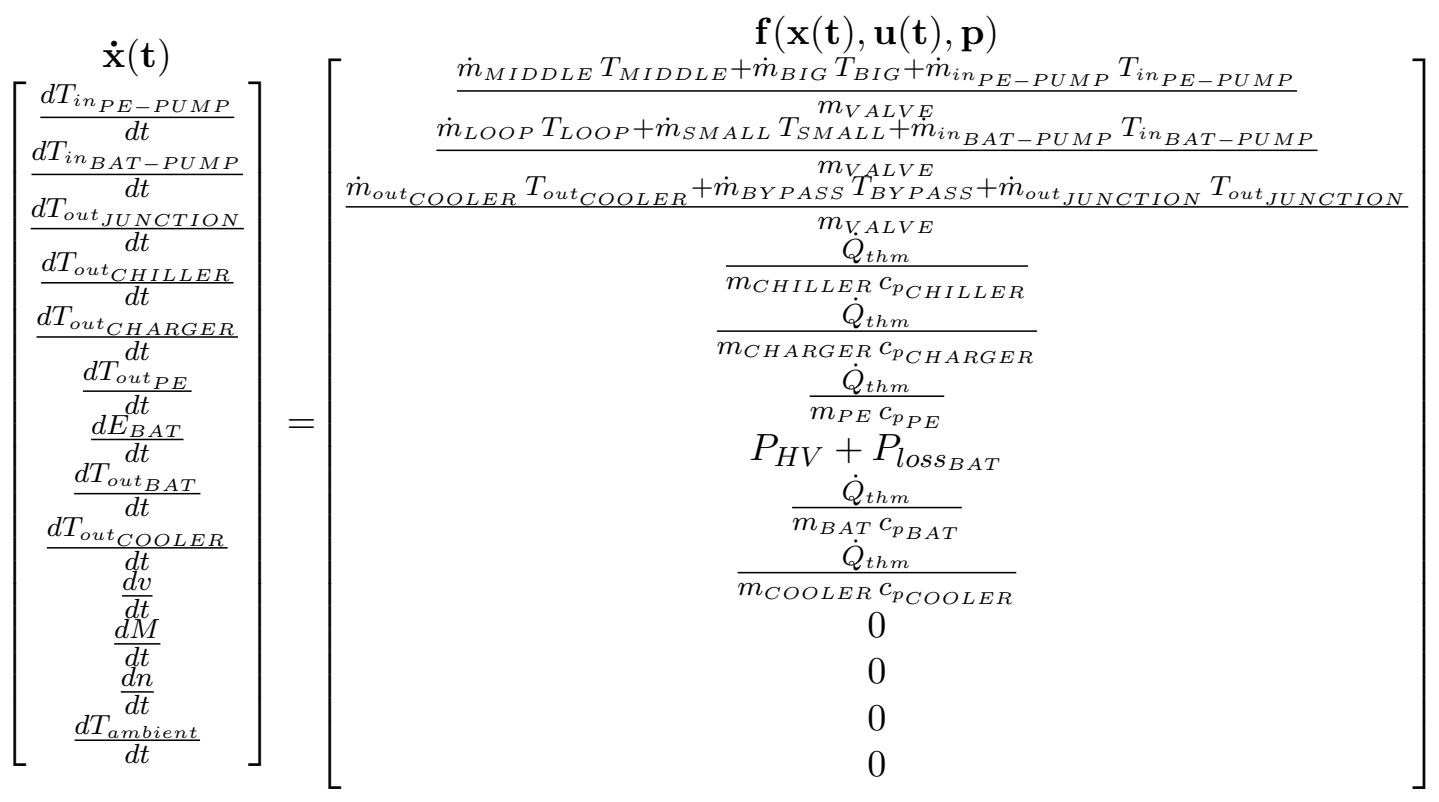

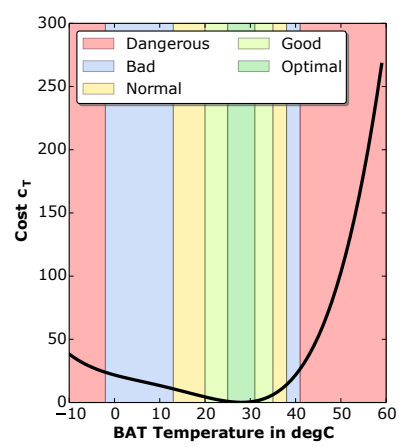

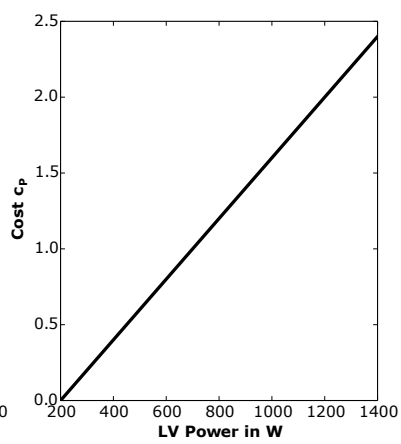

Fig. 4: Cost terms included in the objective function to evaluate accuracy and efficiency of the TM.

optimal range, the more promoted the aging mechanisms, i.e.,

$$
c_{T}(T)=a_{4} T^{4}-a_{3} T^{3}+a_{2} T^{2}-a_{1} T+a_{0},
$$

where $a_{0}, a_{1} \ldots a_{4}$ are the corresponding parameters resultant from the curve fitting. The penalty term $c_{P}$ (on the right of Fig. 4) is the following linear function depending on the electrical power $P$ of the actuators:

$$
c_{P}(P)=\frac{P-b_{0}}{b_{1}},
$$

where again $b_{0}, b_{1}$ are calibration parameters. Besides, $c_{P}$ indicates that the more electrical power is used for the TM, the less attractive it is. Table I shows the electrical actuators used categorizing them according to the amount of electric power they require. The total cost associated to the TM is given by $c$, which is the sum of
TABLE I: Actuators electrical power

\begin{tabular}{lll}
\hline Actuator & Control Signal & $\begin{array}{l}\text { Electrical } \\
\text { power }\end{array}$ \\
\hline Cooler valve & Valve $_{C O O L E R} \in\{0,1\}$ & low \\
Fan & $P W M_{F A N} \in[10,90]$ & high \\
BAT pump & $P W M_{B A T} \in[0,100]$ & medium \\
Chiller valve & Valve $_{C H L L E R} \in\{0,1\}$ & low \\
Compressor & Valve $_{C H L L E R} \in\{0,1\}$ & high \\
Circuit valve & Valve $_{C I R C U I T} \in\{0,1\}$ & low \\
PE pump & $P W M_{P E} \in[0,100]$ & medium \\
\hline
\end{tabular}

the two penalty terms in Fig. 4, i.e.,

$$
c=c_{T}+c_{P} .
$$

Besides the model and objective function, the physical $\quad{ }_{167}$ constraints definition is an important step in the control ${ }_{168}$ problem formulation. Hence, the saturation limits of the 169 control signals, middle column in Table I, were defined 170 as minimal and maximal constraints.

Nevertheless, for the PWM input signals of the pumps, more restrictive minimal constraints were used. They are

$$
\begin{gathered}
\boldsymbol{u}_{\min } \\
{\left[\begin{array}{c}
16 \\
30
\end{array}\right] \leq\left[\begin{array}{c}
P W M_{B A T} \\
P W M_{P E}
\end{array}\right] .}
\end{gathered}
$$

With these restrictive constraints it is assured that a minimal coolant amount flows through the components to protect them from a sudden change in temperature.

Similarly to the control signals, the constraints for the system states are defined as follows: 


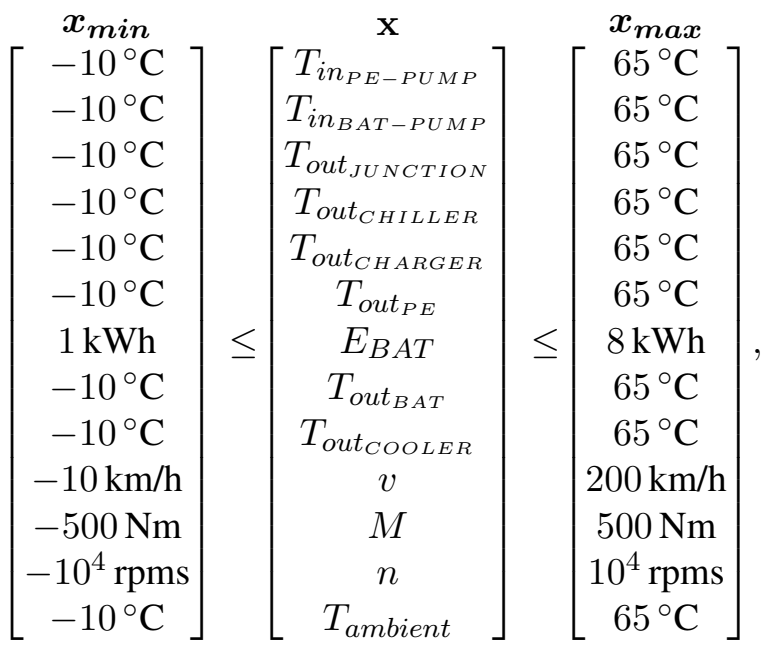

where it must be highlighted that the maximal working temperature for the coolant in this circuit is $65^{\circ} \mathrm{C}$.

With all these requirements, the open-loop finitehorizon optimal control problem (OCP) associated to the cooling circuit can be formulated as follows:

$$
\min _{x^{*}(\cdot), u^{*}(\cdot)} \int_{t_{0}}^{t_{0}+H_{p}}\left(c_{T}+c_{P}\right) d t
$$

subject to

$$
\begin{aligned}
\dot{x}(t) & =f(x(t), u(t), p) & & \forall t \in \tau \\
x_{\text {min }} & \leq x \leq x_{\max } & & \forall t \in \tau \\
u_{\text {min }} & \leq u \leq u_{\max } & & \forall t \in \tau \\
0 & =x\left(t_{0}\right)-x_{0} . & &
\end{aligned}
$$

Given an initial value of the states, $x_{0}$, at time $t_{0}$, the goal of the strategy is to find the optimal sequence of control inputs and states, $u^{*}(\cdot), x^{*}(\cdot)$, that minimizes the objective function in (9a), and satisfy the constraints in (9b-9e), for a given prediction horizon of length $H_{p}$.

Control plant:

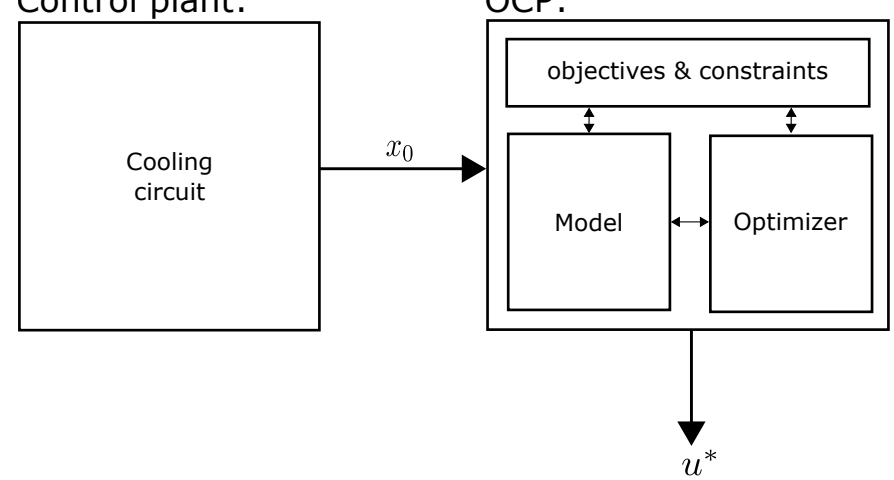

Fig. 5: Optimal control problem outline
To attend model-plant mismatches and overcome possible disturbances, the open-loop scheme in Fig. 5 must be closed resulting in the NMPC scheme in Fig. 6. The main idea behind NMPC is to formulate and solve repetitively a new OCP at each time instant according to the receding horizon strategy. At a certain instant $k$, the measurement of the plant $x$ is used to initialize the ODE with $x\left(t_{0}\right)=x$ used in the constraint (9e) and the OCP is solved to find the optimal control sequence $u^{*}$ for the given prediction horizon. From the solution sequence $u^{*}$, only the first element is applied to the system and the whole procedure is repeated for the next time instant $k+1$ with new sensors measurements coming as the closed-loop system feedback, thus receding the prediction horizon.

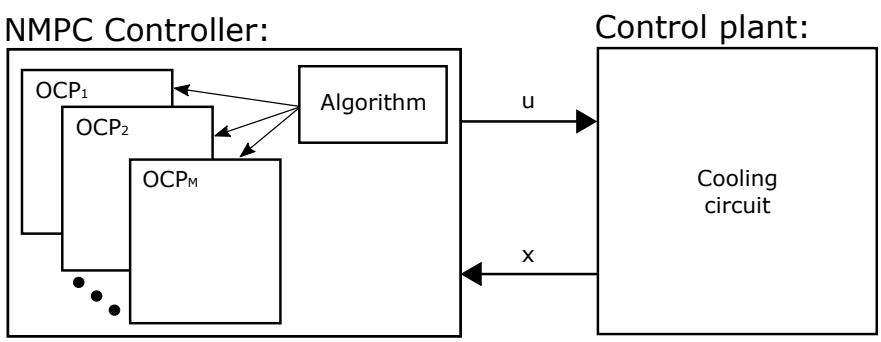

Fig. 6: Control scheme of NMPC

There exist several numerical methods for solving an OCP, as reported in [12]. The optimization tool used in this research, MUSCOD-II, relies on efficient and robust DMS algorithm [13] that reformulates the OCP as a nonlinear programming (NLP) problem that is then solved by an iterative solution procedure, a specially tailored Sequential Quadratic Programming (SQP) algorithm [6]. Notice that the discretization of the continuous optimal control problem is done inside MUSCOD-II. At each time instant, the MSP discretizes the OCP horizon with the following $\mathrm{N}$-points grid:

$$
0=\tau_{0}<\tau_{1}<\ldots<\tau_{N}=t_{f} .
$$

Fig. 7, an example of an optimization horizon of length 201 $t_{f}$ divided in $\mathrm{N}=4$ intervals with five MS points is 202 shown, where it can be seen how one of the thirteen 203 differential states, $x[k]$, and one of the six controls, $u[j] \quad 204$ are discretized according to the MS scheme. 

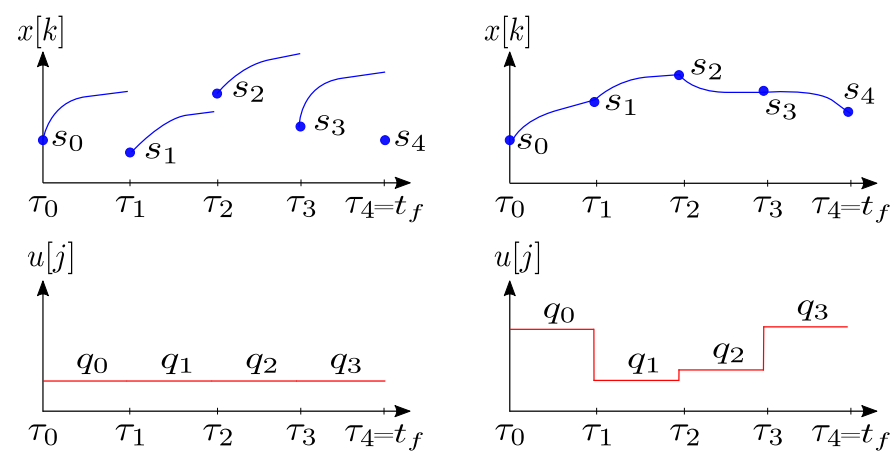

Fig. 7: Multiple shooting method with a grid of 5 shooting points before (left plots) and after (right plots) convergence is achieved.

The left plots in Fig. 7 show the start of the optimization and the right ones belong to the situation once the process has converged. As it can be seen, inside each interval, the controls are parametrized as follows:

$$
u(t):=q_{i}, \quad t \in\left[\tau_{i}, \tau_{i+1}\right)
$$

where $q_{i} \in \mathbb{R}$. Additionally, at each grid point new initial values $s_{i}$ are added. Combining an integrated ODE solver for solving the resulting initial value problems (IVP) and the SQP algorithm, the optimizer searches the controls $q_{0}, q_{1} \ldots q_{N-1}$ and shooting points $s_{0}, s_{1}, s_{2} \ldots s_{N-1}$ that minimize the objective function and fulfill the constraints. In other words, the optimizer solves the following NLP problem:

$$
\min _{\xi} \sum_{i=0}^{N} l_{i}\left(\tau_{i}, s_{i}, q_{i}, p\right)
$$

subject to

$$
\begin{aligned}
s_{i+1} & =x_{i}\left(\tau_{i+1} ; \tau_{i}, s_{i}, q_{i}, p\right) & & 0 \leq i \leq N-1, \\
0 & \leq c\left(\tau_{i}, s_{i}, q_{i}, p\right), & & 0 \leq i \leq N \\
0 & =s_{0}-x_{0} & &
\end{aligned}
$$

where $\xi=\left(q_{0}, q_{1} \ldots q_{N-1}, s_{0}, s_{1}, s_{2} \ldots s_{N-1}\right)$ is a vector with all the unknowns and $x_{i}\left(\tau_{i+1} ; \tau_{i}, s_{i}, q_{i}, p\right)$ denotes the solution of the IVP on the shooting interval $i$, evaluated in $\tau_{i+1}$, and depending on the initial time $\tau_{i}$, initial state $s_{i}$, controls $q_{i}$ and model parameters $p$. The constraint (12b) forces that the trajectory at the end of one interval matches the initial values of the trajectory in the next interval and thus the whole continuity can be assured after convergence is achieved, as it can be seen on the right plots in Fig. 7. Moreover, the constraint (12c) collects the discretized path constraints in (9b)-(9d) while (12d) is the discretized version of $(9 \mathrm{e})$.

Finally, it must be added that MUSCOD-II relies on the so called Real-Time Iteration (RTI) scheme for

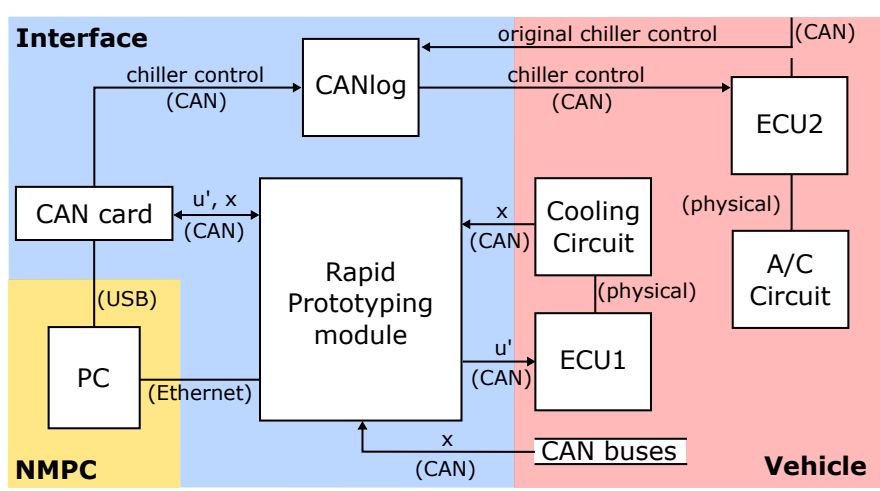

u': pumps, valves and fan control signals

Fig. 8: Hardware implementation for the cooling circuit control manipulation.

achieving robust online performance. The main idea of this algorithm is to exploit the similarity between subsequent OCP for performing the SQP steps in a 226 different order as accustomed, prioritizing this way a 227 fast response time to disturbances. For more information 228 about the RTI scheme, the reader is referred to [14]. ${ }_{229}$ It must be added that the state of the plant, available 230 from several CAN buses, was sampled every $10 \mathrm{~ms}$. ${ }_{231}$ Nevertheless, the communication between the vehicle 232 and MUSCOD-II was asynchronous, being states and 233 controls exchanged as soon as MUSCOD-II performed 234 a new step with the RTI scheme. Using a prediction 235 horizon of 200 seconds and two shooting points, the 236 maximal measured response time of MUSCOD-II was $2.5 \mathrm{~s}$, which is quite acceptable for the studied thermal system inertia.

\section{HARDWARE IMPLEMENTATION}

The PHEV used in this research is a prototype of 241 a Golf GTE equipped with extra sensors placed in the 242 cooling circuit to read all relevant information. In total, 243 17 thermocouples of type $\mathrm{K}$ with accuracy of $\pm 1^{\circ} \mathrm{C}$ were used to measure 15 coolant temperatures, the air temperature in front of the cooler and the air temperature on the roof of the vehicle. In addition, three turbine flow meters with a linearity of $0.1 \%$ were used to measure the coolant volume flow rate.

With the aim of being able to compare the standard control with the NMPC in successive driving tests, the design in Fig. 8 was implemented. With this implementation, it can be switch between two operation modes as explained next.

\section{A. NMPC Mode}

MUSCOD-II runs in the Laptop held by the co-pilot, 256 being connected to a rapid prototyping (RP) module ${ }_{257}$ 235 7

8

29

30
31


.

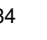


through an Ethernet connection. The control signals are sent by means of the Universal Serial Bus (USB) connected Controller Area Network (CAN) card to the RP module. The electronic control unit (ECU1) is equipped with an emulator test probe (ETK) that allows that the control signals arriving to the ECU1 via the RP ETK connection, are taken instead of the original code in the ECU1 software. This way the original physical electric connections to the actuators in the cooling circuit can be kept. Furthermore, the states of the controlled plant, output signals of the temperature sensors installed in the cooling circuit and other signals running in the CAN buses of the vehicle are sent to MUSCOD-II through the RP module.

Since the chiller valve is physically stimulated from another ECU (ECU2) that is not equipped with ETK, a CAN logger is needed (top right corner of Fig. 8). The CAN logger performs a gateway that splits the CAN bus containing the original command for this valve. This way the Valve $_{C H I L L E R}$ calculated in MUSCOD-II can be used instead of the original vehicle demand.

\section{B. Standard Mode}

The RP deactivates the bypass of ECU1 and the CAN logger sends the signal arriving from the original CAN bus to the ECU2. In this mode, the original control signals of the vehicles for the cooling circuit and AC circuit are taken. These control signals are set to constant values output by a finite-state machine with four possible states: heating, temperature maintaining, mild cooling and maximal cooling. The conditions for changing from one state to another depend on the current BAT temperature and some sensors describing the availability of the heat exchangers to dissipate the heat.

\section{DRIVING SCENARIOS}

A requirement for testing the TM of electric components is to choose a driving cycle in which significant thermal load is generated. This can be achieved with a heavy load cycle driven in the pure electric mode since the heat generated in the components is caused by the Joule Effect. To design a driving cycle with a heavy mechanical demand, three different scenarios were chosen to be performed on an open-accessible street with low traffic density:

- Long cycle mild: A long trip of $39 \mathrm{~km}$ in a road with considerable slope in mild climate conditions.

- Long cycle hot: The same cycle in hot climate conditions.

- Constant cycle: A trip at $100 \mathrm{~km} / \mathrm{h}$ constant speed in a $21 \mathrm{~km}$ road also with considerable slope.
A key aspect of these cycles is the effort put in the design to make them as repeatable as possible. Quite helpful for this task is the adaptive cruise control (ACC) that is available in the car. Other cars are obstacles in the road that prevent the vehicle from following the repeatable cycle forcing the driver to accelerate or break abruptly and therefore they can be considered as external disturbances. Due to the usage of the ACC, these disturbances are held to a minimum since the ACC accelerates and decelerates smoothly, in contrast to the driver natural reaction, thus generates minimal extra load to the battery.

Additionally to achieve always a similar electrical power demand to the BAT, all the tests were driven with the car being under the same conditions. Auxiliary consumers like heating, air conditioning and ventilation (HVAC) were turned off, as well as lights, radio and other electrical gadgets. Windows were opened to the same level and the weight of the car was held the same.

To assure similar initial conditions, it is specially crucial to monitor the BAT temperature before driving, since as it takes direct influence on the objective function, small discrepancies in it will lead to non comparable conditions for the two cycles. Thus, the car is always fully charged the day before in order to assure that all temperatures in the car were close to the ambient temperature and not disturbed by any heat source and that the BAT draws always from with the same energy level. This way, once enough similar conditions are observed, ambient, battery temperatures and traffic congestion, a comparable driving cycle can be assured and the test can start. As it will be seen in the Section VII, this test procedure enabled enough repeatable driving cycles to compare the results of performing a different control in the cooling circuit.

\section{RESULTS}

Experimental results from the three different cycles will be discussed in the following subsections. They are also summarized in Table II, where the consumption, $E$, cost terms, $c_{T}$ and $c_{P}$ and total cost, $c$, are compared for the two operation modes, NMPC and standard, described in Section V. Notice that in Table II a negative value represents a decrease of the cost comparing NMPC to the standard strategy.

\section{A. Long cycle mild}

As it can be seen in the top plot in Fig. 9, where the left y-axis shows the vehicle speed and the right one the altitude of the road, the long cycle consists of a highway road section, in blue, followed by a mountain that is 
TABLE II: NMPC vs standard results in TM for three different driving cycles.

\begin{tabular}{lccccc}
\hline Cycle & $\Delta E^{*}$ in $\mathrm{kWh}$ & $\frac{\Delta \mathbf{E}_{\mathbf{0}}^{* *}}{\mathbf{E}_{\mathbf{o}}}$ in $\%$ & $\frac{\Delta c_{T}}{c_{T_{0}}}$ in $\%$ & $\frac{\Delta c_{P}}{c_{P_{0}}}$ in $\%$ & $\frac{\Delta \mathbf{c}}{\mathbf{c}_{\mathbf{o}}}$ in \% \\
\hline Long cycle mild & -0.015 & $\mathbf{- 6 . 2 5}$ & -8.1 & -20.71 & $\mathbf{- 9 . 9 5}$ \\
Long cycle hot & -0.027 & $\mathbf{- 8 . 1 4}$ & -54.26 & -17.12 & $\mathbf{- 5 0 . 0 4}$ \\
Constant cycle & 0.003 & $\mathbf{3 . 4 9}$ & -8.2 & 5.09 & $\mathbf{- 7 . 7 8}$ \\
\hline
\end{tabular}

" $\Delta x$ stands for the measured difference in the value " $\mathrm{x} ": x_{N M P C}-x_{\text {Standard }}$.

${ }^{* *} x_{0}$ stands for the measured value " $\mathrm{x}$ " in the Standard cycle: $x_{\text {Standard }}$.
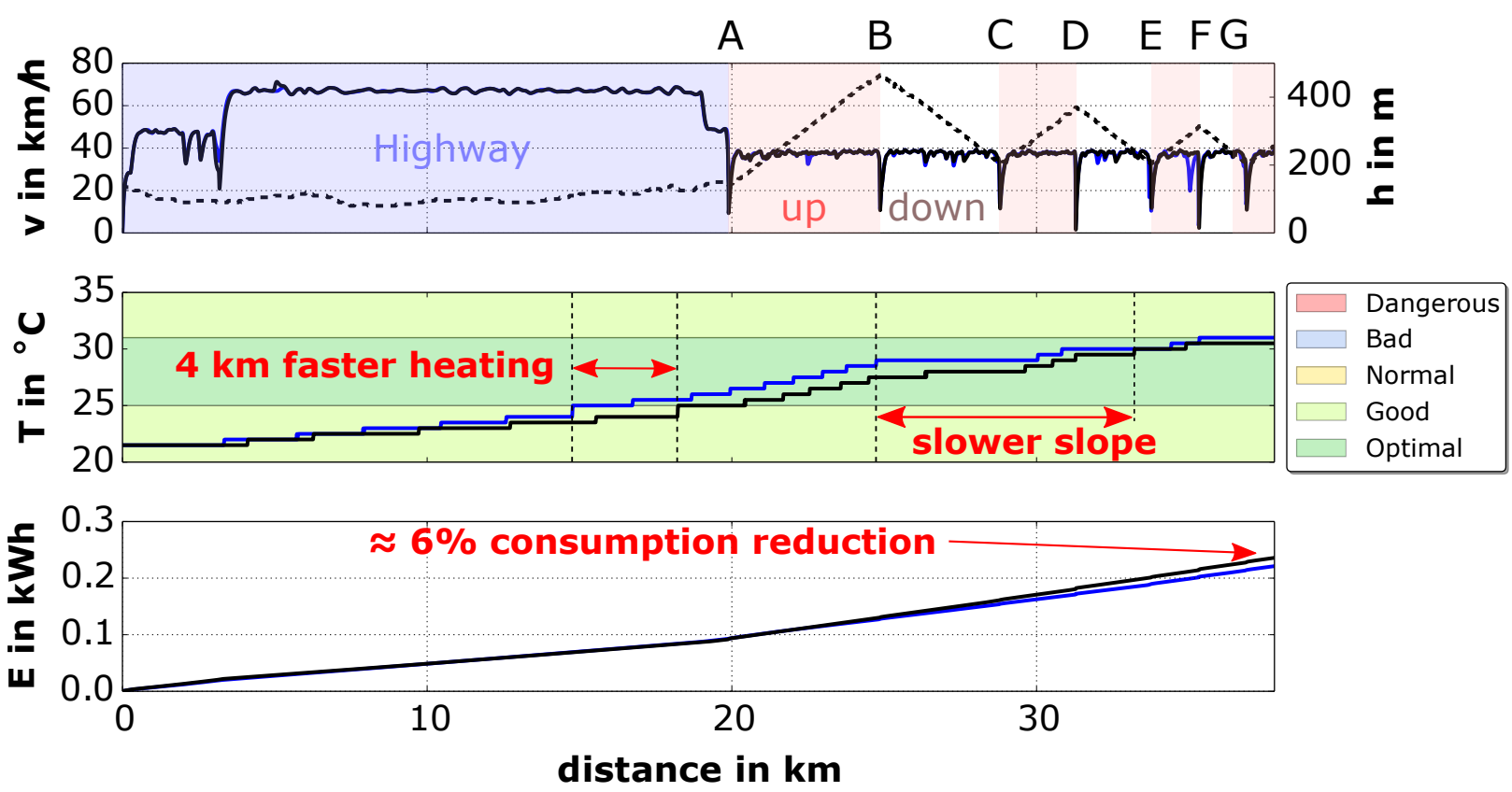

NMPC - Standard

Fig. 9: NMPC vs standard TM results in terms of temperature accuracy and electrical consumption of the actu in the long cycle mild.

ascended to the top, in red, discharging the battery and then descended to the bottom, in white, charging the battery again.

The aim of this driving cycle is to keep the BAT working and thus generating heat as much time as possible under heavy conditions. To achieve this, in the slope road section several strategic turning points were predefined. This way, the vehicle faces the slope for the first time at $\mathrm{A}$ and drives till the highest point $\mathrm{B}$ is reached, where the vehicle turns over and starts the descent to the initial kilometric point $\mathrm{A}$, now named $\mathrm{C}$ in Fig. 9. Again, the vehicle turns over and drives to the next turning point, D, lower than B and so on till, after the last turn over in G, the BAT is fully discharged and the pure electric mode is no longer available. The small variations in the speed profile during NMPC (blue solid line) and standard control (black solid line) allow to assume that the results discussed draw from comparable conditions.

In the middle and bottom plots in Fig. 9, the TM resulting from the NMPC and standard strategies in a mild thermal scenario, ambient temperatures around $14^{\circ} \mathrm{C}$ and initial BAT temperature $22^{\circ} \mathrm{C}$, can be compared. Concerning the goal of keeping the battery within optimal temperatures, it can be seen in the middle plot that NMPC reaches the optimal range about $4 \mathrm{~km}$ faster than the standard control strategy. Once inside this range, the slope decreases to maintain the BAT at this level. Moreover, the second goal, the electric consumption shown in the plot on the bottom, is reduced by $6 \%$. The NMPC success in multiple objective achievements can be seen in detail in Fig. 10. Focusing on the temperature and consumption related costs of NMPC, blue line in the top and middle plot in Fig. 10, respectively, three differentiated strategic phases for the control can be derived: 1) Battery heating phase (blue area in Fig. 10) in 




Fig. 10: NMPC vs standard objective function costs.

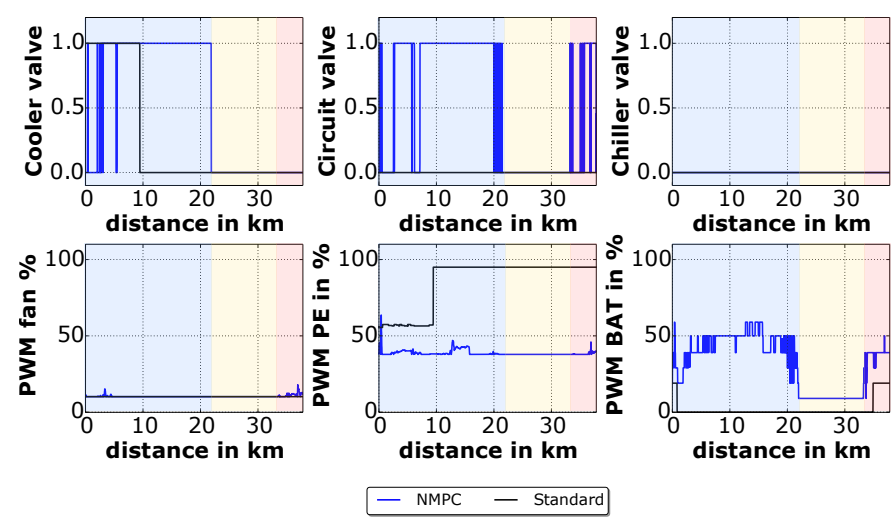

Fig. 11: NMPC vs standard control strategies in the long cycle mild.

which the main goal is to bring the battery temperature to the optimum as it is shown in the top plot with the faster decrease of the temperature cost in NMPC inside the blue area. The prize to pay is a slightly higher electrical consumption as represented in the middle plot, 2) Energy saving phase (yellow area in Fig. 10) where the priority is to minimize the actuators electrical consumption as it can be seen clearly in the yellow area of the middle plot and 3) Battery cooling phase (red area in Fig. 10) in which the temperature costs, this time associated to higher temperatures than the optimal, are again high enough to invest resources. Inside the different described phases, the control inputs from the NMPC strategy show a tendency as it can be seen in Fig. 11.

For heating the BAT, the cooler valve is bypassed and the circuit valve enables the big circuit mode that couples the BAT and the PE. As Fig. 12 shows, this is a clever way to heat the BAT since compared to it, the PE has a higher temperature and the air flowing through the cooler a lower one.

Once the optimal temperature is achieved, as shown in Fig. 12, the cooler is activated as well as the two circuit mode. The BAT is decoupled from the PE at

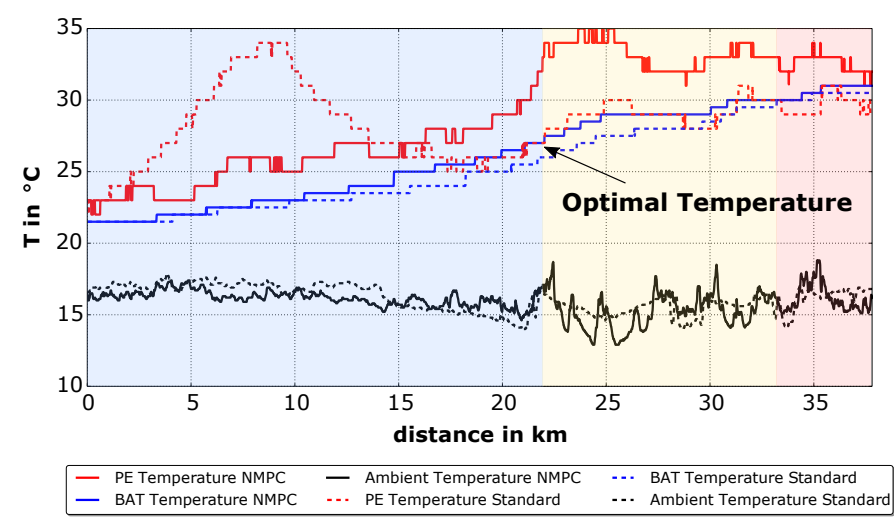

Fig. 12: NMPC vs standard components and ambient temperatures in the long cycle mild.

this moment, because the PE is warmer and the BAT ${ }_{416}$ is already at its optimal temperature. The reason for the ${ }_{417}$ cooler activation is to dissipate to the air the heat that ${ }_{418}$ is being generated in the PE module due to the road 419 slope. This way, the constraint of not exceeding $65^{\circ} \mathrm{C}$ in this module is achieved. It must also be said that, in this phase (yellow area), the battery pump is brought down to its minimum in order to save energy. As soon as the BAT temperature starts deviating from the optimal one, about $3^{\circ} \mathrm{C}$, the circuit valve enables and disables the coupling to the PE circuit intermittently.

\section{B. Long cycle hot}

The same cycle was driven under hotter conditions having been the vehicle parked outdoors, exposed to direct sunlight: average ambient temperature around $20^{\circ} \mathrm{C}$ and initial BAT temperature $31-31.5^{\circ} \mathrm{C}$. Again, despite some punctual speed discrepancies due to different traffic situations, the cycles in Fig. 13 are enough similar to be compared.

As it can be seen in Table II, in this cycle there is even more potential than in the mild climate case. The consumption is reduced this time by $8 \%$ while the temperature trajectory is more accurate, temperatures closer to the optimal range, than with the standard control. The combination of these two goals leads to a numerical improvement of $50 \%$ in the objective function. In general, it can be said that the more cooling requiring the situation is, the more potential NMPC has. This is due to the fact that the studied cooling circuit has several heat sinks for actively cooling the components but no heat sources for heating the battery. That means that under cold conditions, the only possibility is to take advantage from the different inertias of the components in the system while under hot conditions, the many cooling alternatives lead to completely different results.
418 420 421 422 423 424 425 426 

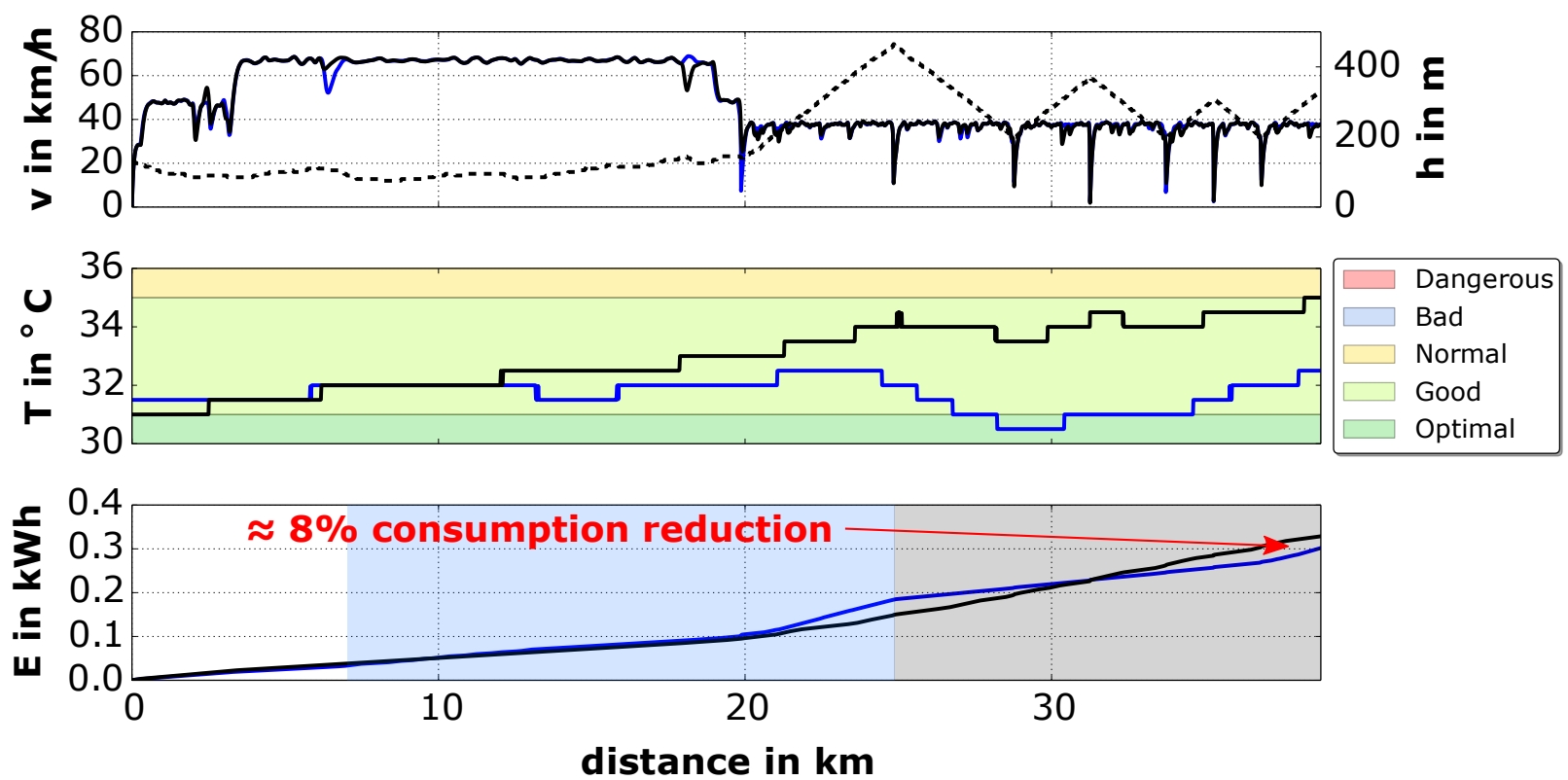

distance in $\mathbf{k m}$

Fig. 13: NMPC vs standard TM results in terms of temperature accuracy and electrical consumption of the actuators in the long cycle hot.



Fig. 14: NMPC vs standard objective function costs in the long cycle hot.
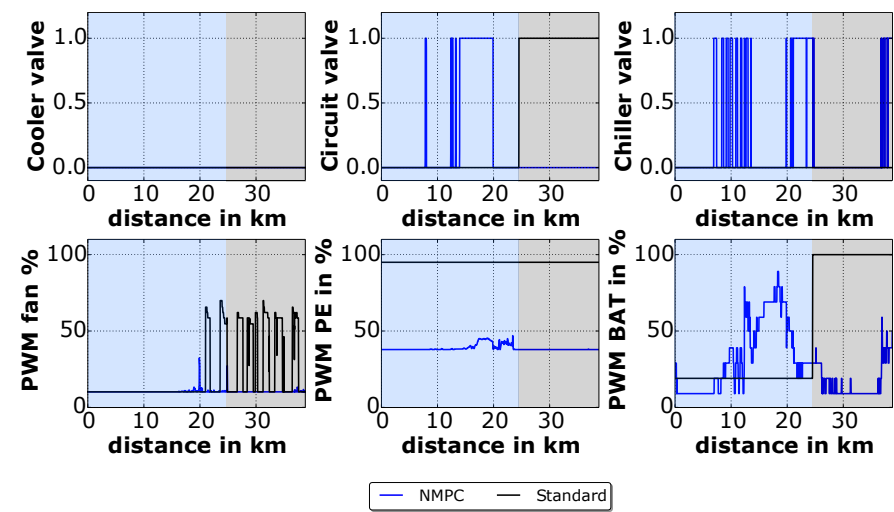

Fig. 15: NMPC vs standard control strategies in the long cycle hot.

soon as the BAT temperature is too far from the optimum 464 it uses the pumps at full and the fan at medium power. 465

In Fig. 16 the BAT, PE and ambient temperatures 466 for both cycles are compared. Although the ambient ${ }_{467}$ temperature at the end of the cycle, last $25 \mathrm{~km}$, is lower 468 in the standard cycle, the NMPC strategy achieves a ${ }_{469}$ more accurate regulation of the BAT temperature. Notice 470 also that both PE curves are far away from the critical 471 temperature of $65^{\circ} \mathrm{C}$ for the component, imposed in the 472 NMPC case by means of a constraint.

While NMPC invests in the chiller and moderately in the pumps in an intermittent way to cool down the BAT temperature, the standard control strategy shows two clearly differentiated working points: previous to the black region, it only uses the PE pump and the cooler valve to cool down the $\mathrm{PE}$ and inside the black region, as 




Fig. 16: NMPC vs standard components and ambient temperatures in the long cycle hot.

\section{Constant cycle}

The constant driving cycle consists of the entrance to the highway, first $4 \mathrm{~km}$ in Fig. 17, and then the drive on the highway at constant speed of $100 \mathrm{~km} / \mathrm{h}$. The highway road has a considerable slope that, together with the high speed, leads to the full discharge of the BAT in the 17 minutes duration of the whole cycle. Again, the TM with the NMPC presents a decrease in the global cost function $c$ of Table II compared to the standard control. Although, the electrical consumption of the actuators is increased by $3.5 \%$, as it can be seen in Fig. 17, the faster heating of the BAT to the optimal temperature compensates this loss.

One of the main reasons for these results being less attractive than in the other driving cycles is that this one starts at colder temperatures, the initial BAT temperature is $14^{\circ} \mathrm{C}$, and thus the potential of the system is reduced. The cooling circuit has several options for cooling the BAT, the cooler and chiller, but for generating heat it can only wait to use the heat generated in the PE, which has a lower thermal mass.

As shown in Fig. 18 and in contrast to the costs within the long cycle in Fig. 10, here NMPC follows nearly all the cycle long the same strategy, to reduce the penalty term $c_{T}$. Only at the end, after $20 \mathrm{~km}$, it starts to play with the chiller valve as shows the red arrow in Fig. 18.

It must be added that the fact that this cycle is driven at constant speed, places the standard strategy in an advantageous situation, since finite-state machines are experience is available. Therefore, the less transient and the more common the driving conditions are, the more accurate is this method. In this case, the standard finitestate machine shows two fixed operation points as it can be seen with the black solid lines in Fig. 19.

Moreover, it must be added that the last $5 \mathrm{~km}$ of this usually defined with several static points at which control cycle are not as comparable as desired, since as it is 510 shown in Fig. 20 the ambient temperature in the NMPC 511 case is around $3^{\circ} \mathrm{C}$ above the standard control case. ${ }_{512}$ This fact could be an extra disadvantage for the NMPC ${ }_{513}$ since this happened when the BAT was already close to 514 the optimal temperature and hence the cooling potential 515 through the air is less. Furthermore, the presence of some 516 traffic before ending the cycle, as it can be seen in Fig. ${ }_{517}$ 17 , leads to a more abrupt deceleration and thus to a 518 higher heat generation in NMPC, being this a further disadvantage at temperatures close to the optimal, as it is the case.

All in all, it can be said that even in an scenario where the standard control strategy can show its major performance, the NMPC still achieves a more accurate TM. It must be also said that the fact that one goal, the electrical consumption, becomes worse in favor of the other goal, temperature regulation, is a mere strategic matter. One of the advantages of the proposed NMPC strategy is that terms in the objective function, $c_{T}$ and $c_{P}$, can be changed or modified to achieve other results. Compared to a PID tuning method, this calibration is simpler since the parameters adjusted have a physical meaning whose effect on the goals can be reproduced and observed with a limited number of experiments or simulations.

\section{CONCLUSIONS}

In this paper, a real-time NMPC for the Li-ion battery and power electronics cooling circuit in a PHEV prototype has been validated with three different repeatable driving cycles performed on the road. In all studied cases, NMPC has shown a significant decrease, from $7 \%$ up to $50 \%$, in the total costs associated to an accurate and efficient TM when compared to a standard control strategy based on a finite-state machine.

Analyzing the results according to the two objectives separately, it can be said that the temperature cost was reduced in the three studied cases while the electrical consumption was reduced, between 6 and $8 \%$, only in the long cycle tests. In the constant cycle it was increased by $3.5 \%$. Although the overall cost for this cycle is already satisfactory, if additionally both goals should be improved at the same time, it would be quite straightforward to achieve adjusting the cost functions. This is a further advantage in comparison with a PID tuning process where the effect of the $\mathrm{P}, \mathrm{I}$ and $\mathrm{D}$ gains on the several goals are not so intuitively and directly attributable to them.

This may seem paradoxical, but there are two reasons for the constant cycle presenting the most moderate improvement of the three cycles. On the one hand, the cold 10

4

15

516





$\mathrm{H}$

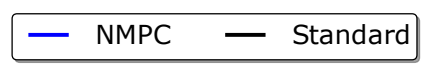

Fig. 17: NMPC vs standard TM results in terms of temperature accuracy and electrical consumption of the actuators in the constant cycle.

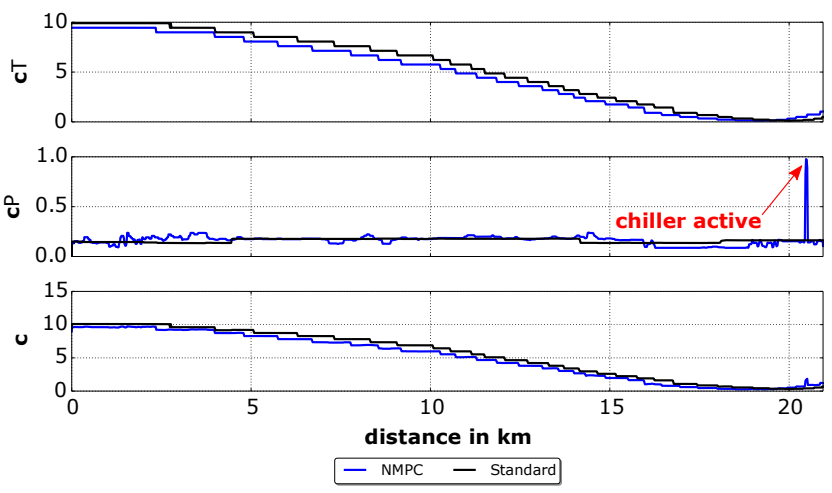

Fig. 18: NMPC vs standard objective function costs in the constant cycle.

temperatures in this cycle reduce considerably the potential of the control strategy because the studied cooling circuit cannot generate any other heat than the induced by the Joule Effect. On the contrary, in a hot scenario as in the long cycles studied, the heat dissipation can be done to the ambient air or to the A/C circuit through the several actuators, thus leading to many control options for cooling the components. Therefore, it can be said that under complex situations with many control options NMPC methods show the highest potential. On the other hand, the untapped potential of the standard strategy is reduced in a quite steady cycle such as the constant cycle, because finite-state machine are usually designed using measured data at several stationary points. Nevertheless,
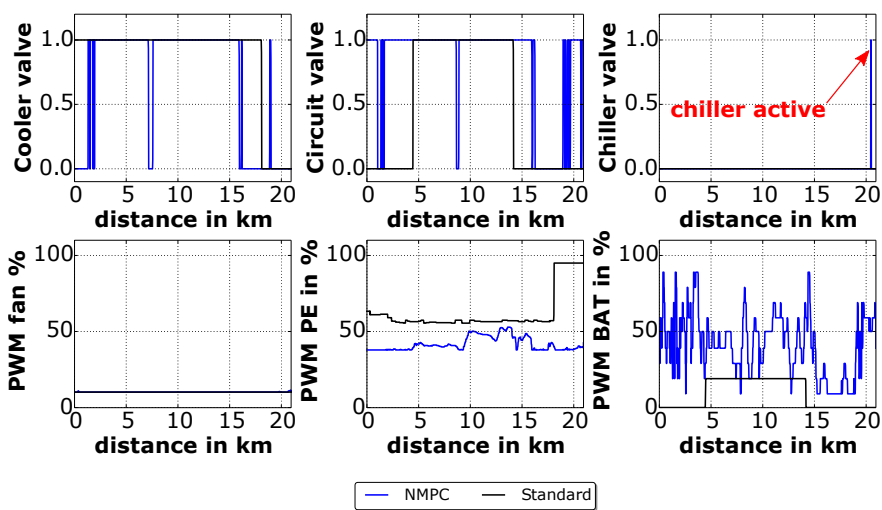

Fig. 19: NMPC vs standard control strategies in the constant cycle.

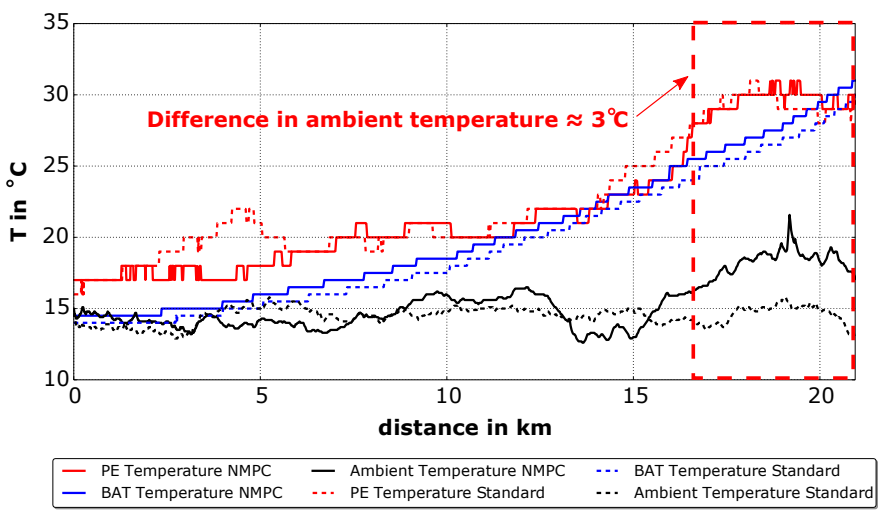

Fig. 20: NMPC vs standard components and ambient temperatures in the constant cycle. 
it was shown that even in this situation, the NMPC is able to grasp part of the untapped potential of the standard strategy.

Finally, it must be concluded that the OCP formulated, by means of a simple and accurate model, and the DMS and RTI algorithm implemented in MUSCOD-II, have led to an NMPC control strategy that has shown a stable and real-time capable performance. Future works will be focused on the improvement through the use of a driving cycle prediction and the mixed-integer optimal control problem (MIOCP) formulation and solution.

\section{REFERENCES}

[1] A. A. Pesaran, "Battery Thermal Management in EVs and HEVs : Issues and Solutions," in Advanced Automotive Battery Conference, Las Vegas, Nevada, 2001.

[2] R. Schmied, H. Waschl, R. Quirynen, and M. Diehl, "Nonlinear MPC for Emission Efficient Cooperative Adaptive Cruise Control," in 5th IFAC Conference on Nonlinear Model Predictive Control, 2015.

[3] N. V. Duijkeren, T. Keviczky, and P. Nilsson, "Real-Time NMPC for Semi-Automated Highway Driving of Long Heavy Vehicle Combinations," in 5th IFAC Conference on Nonlinear Model Predictive Control, 2015.

[4] Z. Jiangyan, S. Tielong, S. Takanobu, and K. Masaaki, "Nonlinear MPC-Based Power Management Strategy for Plug-in Parallel Hybrid Electrical Vehicles," in Proceedings of the 33rd Chinese Control Conference, 2014.

[5] J. Zhao, S. Member, J. Wang, and S. Member, "Integrated Model Predictive Control of Hybrid Electric Vehicles Coupled with Aftertreatment Systems," IEEE Transactions on Vehicular Technology, vol. 9545, no. Not published yet, but accepted for a future issue., pp. 1-13, 2015.

[6] C. Hoffmann, L. Wirsching, M. Diehl, D. B. Leineweber, A. A. S. Sch, H. G. Bock, and J. P. Schl, "MUSCOD-II user manual," Heidelberg, 2010.

[7] H. Chen, S. Yu, X. Lu, F. Xu, T. Qu, and F. Wang, "Applying Model Predictive Control in Automotive," in Proceedings of the 10th World Congress on Intelligent Control and Automation, 2012.

[8] J. L. Sanz, C. Ocampo-Martinez, J. Alvarez-Florez, M. M. Eguilaz, R. Ruiz-Mansilla, J. Kalmus, M. Graber, and G. Lux, "Nonlinear Model Predictive Control for Thermal Management in Plug-in Hybrid Electric Vehicles," IEEE Transactions on Vehicular Technology, vol. PP, no. 99, p. 1, 2016.

[9] D. AB, "Dymola Users Manual Version 5.3a," Lund, Sweden, 2004. [Online]. Available: http://www.dynasim.com

[10] M. Association, "Modelica - A Unified Object-Oriented Language for Physical Systems Modeling Language Specification," Modelica Association, Tech. Rep., 2010. [Online]. Available: http://www.modelica.org

[11] M. Gräber, C. Kirches, D. Scharff, and W. Tegethoff, "Using Functional Mock-up Units for Nonlinear Model Predictive Control," in Proceedings of the 9th International Modelica Conference, Munich, 2012.

[12] M. Diehl, H. J. Ferreau, and N. Haverbeke, "Efficient Numerical Methods for Nonlinear MPC and Moving Horizon Estimation," in Nonlinear Model Predictive Control, L. et al Magni, Ed. Springer, 2009, pp. 391-417.

[13] H. G. Bock and K. J. Plitt, "A multiple shooting algorithm for direct solution of optimal control problems," in Proceedings 9th IFAC World Congress Budapest, vol. XLII, 1984, pp. 243-247.
[14] M. Diehl, H. G. Bock, J. P. Schlo, R. Findeisen, 636 Z. Nagy, and F. Allgo, "Real-time optimization and 637 nonlinear model predictive control of processes governed 638 by differential-algebraic equations," Journal of Process 639 Control, vol. 12, pp. 577-585, 2002. [Online]. Available: www.elsevier.com/locate/jprocont 